\title{
Visualization of Flavor of Sake by Sensory Evaluation and Statistical Method
}

\author{
Yoichiro Kanno, ${ }^{1,2 *}$ Toshitaka Minetoki, ${ }^{1}$ Takayuki Bogaki, ${ }^{1}$ and Kiyoshi Toko ${ }^{2}$ \\ ${ }^{1}$ Ozeki Corporation, 4-9 Imazu Dezaike, Nishinomiya, Hyogo 663-8227, Japan \\ ${ }^{2}$ Kyushu University, Graduate School of Information Science and Engineering, Kyushu University, \\ 744 Motooka, Nishi-ku, Fukuoka, Fukuoka 819-0395, Japan
}

(Received October 19, 2017; accepted December 14, 2017)

Keywords: taste sensor, sake, sake flavor, sensory evaluation, statistical method

Sake brewing is a complicated process. Different kinds of sake with various tastes are produced depending on the raw materials [e.g., rice, water, rice malt (koji), and sake yeast (kobo)], brewing process, and storage period. To represent the complicated taste of sake in an easy-tounderstand manner, two-dimensional charts based on the analysis results of taste and aroma components have been developed. However, various issues related to the difference between the evaluations by panelists in actual sake tasting and those in charts as well as the lack of objectivity still remain. The purpose of this study is to visualize the taste of sake using a scale that is different from the conventional one by combining taste sensors and sensory evaluation. In this study, measurement using taste sensors, general analysis, and sensory evaluation of 135 kinds of sake were carried out. Using the results obtained, a scatter diagram of sake samples with small differences in the evaluations by panelists in actual sake tasting and those in charts was developed using saltiness determined with taste sensors and a sake meter value (specific gravity of sake). We called the scatter diagram the sake flavor chart. From the obtained results, we succeeded in visualizing the flavor of sake using a new objective scale that faithfully simulates the human sense of taste.

\section{Introduction}

Sake brewing is a complicated process. Different kinds of sake with various tastes are produced depending on the raw materials [e.g., rice, water, rice malt (koji), and sake yeast (kobo)], brewing process, and storage period. ${ }^{(1)}$ To represent the flavor of sake, various methods of analyzing taste components, such as amino and organic acids, and aromatic components, such as ethyl hexanoate and isoamyl acetate called ginjo fragrance, have been established. ${ }^{(2)}$ Furthermore, various organizations have released two-dimensional charts that represent the taste of sake in an easy-to-understand manner. ${ }^{(3,4)}$ However, because of the absence of a clear standard for such representation, charts developed by different organizations differ. Furthermore, various issues, including the difference between the evaluations by panelists in actual sake tasting and those in charts, that is, the lack of objectivity, still remain. 
In fact, in interviews with general consumers in retail stores, the following responses were obtained: "I do not know which sake matches my taste", "I cannot find my favorite sake because there is no clear standard for sake". (5)

With such a background, we focused on taste sensors that can objectively evaluate the flavor of food. These taste sensors have artificial lipid membranes simulating human tongues and have been applied to the measurement of various food products as a device that can objectively and quantitatively evaluate taste. ${ }^{(6-9)}$ Objective numerical data on sake flavor are considered to be obtainable using taste sensors. Furthermore, the quality of sensory evaluation by human panelists, which is the most common conventional method of assesssing sake, was also reexamined.

The purpose of this study is to visualize the flavor of sake using a scale different from the conventional one through the combined use of taste sensors and sensory evaluation. When sake taste can be objectively represented by a scale that is consistent with that determined by sensory evaluation, the scale can be used as a new method for tasting sake in the future.

\section{Materials and Methods}

\subsection{Sake samples used in evaluation}

In this study, 135 kinds of commercially available sake were used (Table 1).

\subsection{Taste sensor}

The taste sensing system TS-5000Z (Intelligent Sensor Technology, Inc.) was developed as a system for quantitatively evaluating taste; it has recently been applied to the analyses of various food products. The taste sensing system consists of five taste sensors corresponding to five basic tastes. Each taste sensor has an artificial lipid membrane that reacts with a particular taste component. The potential of the artificial lipid membrane changes when a taste component reacts with the membrane and is converted into a numerical value that represents the intensity of each basic taste. ${ }^{(10-12)}$

Table 1

Sake samples.

\begin{tabular}{|c|c|c|c|c|c|c|c|c|}
\hline $\begin{array}{l}\text { Sake } \\
\text { brewery }\end{array}$ & Sample & Classification & & & Alcohol & $\begin{array}{c}\text { Sake meter } \\
\text { value }\end{array}$ & Total acid & Amino acid \\
\hline \multirow{8}{*}{48} & \multirow{8}{*}{135} & Regular Sake & Nama Chozo & $\max$. & 17.8 & 8.2 & 3 & 3.1 \\
\hline & & Honjozo & Nama & $\min$. & 6.9 & -59.8 & 0.85 & 0.35 \\
\hline & & Junmai & Conventional & & & & & \\
\hline & & Junmai Ginjo & & & & & & \\
\hline & & Junmai Daiginjo & & & & & & \\
\hline & & Ginjo & & & & & & \\
\hline & & Daiginjo & & & & & & \\
\hline & & Sparkling & & & & & & \\
\hline
\end{tabular}




\subsection{General items evaluated}

The sake meter value (specific gravity), total acid, amino acid level, and alcohol percentage of sake samples were measured. The measurement was carried out in accordance with the prescribed method of the National Tax Agency.

In the measurement of taste using the taste sensing system, control samples are necessary to relatively evaluate the tastes of many samples. A synthesized sake sample with taste components similar to those of general sake was prepared (Table 2). Referring to the synthesized sake as the control, the values of sake samples were relatively evaluated.

\section{Results and Discussion}

\subsection{Development of two-dimensional scatter diagram}

From the data of sake obtained using various analytical devices, the correlation among the items evaluated and the variance of their values were calculated. The results indicate certain relationships among some of the items evaluated (Table 3): a very high negative correlation between sourness and umami; correlations between alcohol percentage and saltiness, alcohol percentage and umami, and alcohol percentage and sourness; high correlations between the sake meter value and umami, and sake meter value and sourness; and high correlations between amino acid level and umami, amino acid level and sourness, and amino acid level and saltiness.

Table 2

Synthetic sake recipe.

\begin{tabular}{lc}
\hline Reagents & $/ \mathrm{L}$ \\
\hline Monosodium glutamate (MSG) & $200 \mathrm{mg}$ \\
Disodium succinate & $600 \mathrm{mg}$ \\
L(-)-malic acid & $300 \mathrm{mg}$ \\
Lactic acid & $400 \mu \mathrm{L}$ \\
D(+)-glucose & $30 \mathrm{~g}$ \\
Calcium sulfate dihydrate & $200 \mathrm{mg}$ \\
Taste sensor [bitterness (-) sample] & $50 \mathrm{ml}$ \\
EtOH (99.5\%) & $150 \mathrm{ml}$ \\
\hline
\end{tabular}

Table 3

Relationships among measurement components.

\begin{tabular}{|c|c|c|c|c|c|c|c|c|c|c|c|c|}
\hline & Sourness & Bitterness & Astringency & Umami & Saltness & $\begin{array}{c}\text { CPA } \\
\text { (bitterness) }\end{array}$ & $\begin{array}{c}\text { CPA } \\
\text { (astringency) }\end{array}$ & $\begin{array}{c}\text { CPA } \\
\text { (umami) }\end{array}$ & Alcohol & $\begin{array}{c}\text { Sake meter } \\
\text { value }\end{array}$ & $\begin{array}{l}\text { Total } \\
\text { acid }\end{array}$ & $\begin{array}{c}\text { Amino } \\
\text { acid }\end{array}$ \\
\hline Sourness & 1.00 & & & & & & & & & & & \\
\hline Bitterness & -0.16 & 1.00 & & & & & & & & & & \\
\hline Umami & -0.94 & 0.13 & -0.07 & 1.00 & & & & & & & & \\
\hline Saltness & -0.48 & 0.03 & 0.00 & 0.70 & 1.00 & & & & & & & \\
\hline CPA (umami) & -0.54 & 0.13 & 0.08 & 0.58 & 0.52 & -0.06 & 0.24 & 1.00 & & & & \\
\hline Alcohol & -0.43 & 0.32 & 0.09 & 0.67 & 0.77 & 0.29 & 0.23 & 0.52 & 1.00 & & & \\
\hline Sake meter value & -0.47 & 0.26 & 0.19 & 0.54 & 0.31 & 0.04 & 0.31 & 0.35 & 0.60 & 1.00 & & \\
\hline Total acid & 0.13 & -0.25 & -0.12 & -0.11 & -0.09 & 0.05 & -0.18 & -0.04 & -0.11 & -0.16 & 1.00 & \\
\hline Amino acid & -0.52 & 0.24 & 0.17 & 0.56 & 0.60 & -0.07 & 0.27 & 0.56 & 0.29 & -0.05 & 0.05 & 1.00 \\
\hline
\end{tabular}

CPA: Change in electric Potential due to Absorption of chemical substances onto the membrane 
The variance of the results obtained differed among the items evaluated. The variances of sourness, saltiness, and sake meter value were relatively high (Table 4).

On the basis of the above results, a two-dimensional scatter diagram of two items selected from the items evaluated was developed. Considering the correlation coefficients and variances obtained, a combination of two items that had high correlation coefficients with other items and showed the largest distribution on the scatter diagram was selected. As a result, the combination of saltiness measured with the taste sensing system and sake meter value was selected because it produced a scatter diagram with the largest distribution. The ordinate and abscissa of the scatter diagram were sake meter value and saltiness, respectively.

However, the center of variance of the obtained scatter diagram deviates from the medians. Thus, compensation along each axis was carried out using the following equations:

$$
\text { Values on ordinate }=\text { Sake meter value }-3,
$$

$$
\text { Values on abscissa }=\text { Saltiness }- \text { Sake meter value } 10-1.5 .
$$

By applying Eqs. (1) and (2), the median of the values of the samples approaches the center of the scatter diagram. Thus, a two-dimensional scatter diagram showing the flavor of sake using a scale that is different from the conventional one and which was based on an objective evaluation standard for the results obtained using analytical devices was successfully developed (Fig. 1).

Table 4

Variances of measurement components.

\begin{tabular}{lrcrrrrrrrrrr}
\hline & Sourness & Bitterness & Astringency & Umami & Saltness & $\begin{array}{c}\text { CPA } \\
\text { (bitterness) }\end{array}$ & $\begin{array}{c}\text { CPA } \\
\text { (astringency) }\end{array}$ & $\begin{array}{c}\text { CPA } \\
\text { (umami) }\end{array}$ & $\begin{array}{c}\text { Alcohol } \\
\text { Sake meter } \\
\text { value }\end{array}$ & $\begin{array}{c}\text { Total } \\
\text { acid }\end{array}$ & $\begin{array}{c}\text { Amino } \\
\text { acid }\end{array}$ \\
\hline Distribution & 6.88 & 1.24 & 0.21 & 1.98 & 4.99 & 0.06 & 0.02 & 0.07 & 2.85 & 86.96 & 1.44 & 0.19 \\
Average & -0.36 & 0.49 & 0.29 & -0.03 & 0.74 & 0.13 & 0.06 & 0.08 & 15.19 & 0.80 & 1.54 & 1.21 \\
Standard deviation & 2.62 & 1.11 & 0.46 & 1.41 & 2.23 & 0.24 & 0.14 & 0.26 & 1.69 & 9.33 & 1.20 & 0.43 \\
Coefficient of variation & -7.34 & 2.25 & 1.58 & -43.04 & 3.01 & 1.94 & 2.23 & 3.51 & 0.11 & 11.64 & 0.78 & 0.35 \\
\hline
\end{tabular}

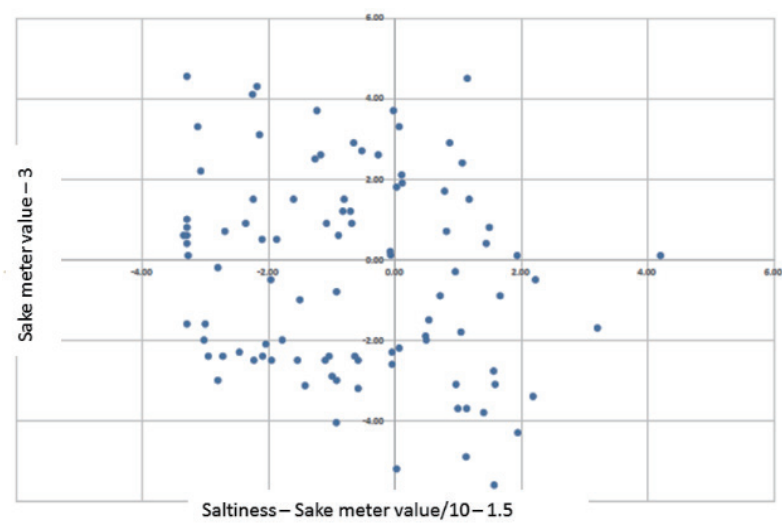

Fig. 1. (Color online) Two-dimensional scatter diagram showing the taste of sake. Ordinate: Sake meter value - 3; Abscissa: Saltiness - Sake meter value/10 - 1.5. 


\subsection{Sensory evaluation}

The axes of the two-dimensional scatter diagram were those for the numerical data of saltiness and sake meter value. Whether or not these numerical data correspond to sensory evaluation data by human panelists has not yet been demonstrated. We then gave meaning to the two-dimensional scatter diagram by combining the scatter diagram with the sensory evaluation data.

The sensory evaluation was carried out by qualitative descriptive analysis (QDA). ${ }^{(13)}$ First, ten points (corresponding to samples A-J) were extracted (Fig. 2) from the scatter diagram. Six trained panelists who are highly skilled in tasting sake were selected from among employees of Ozeki Corporation. They were asked to taste 10 sake samples and evaluate them using the following 13 evaluation words.

- Marumi (fullness with little irritation)

- Fukurami (widening taste in the mouth)

- Koku (robust and rich taste)

- Atsumi (deep taste)

- Sakiaji (strong initial taste)

- Atoaji (aftertaste)

- Odayaka (balanced taste)

- Soft (soft taste)

- Keikai (light taste)

- Sarari (not sticky but smooth)

- Sukkiri (fresh initial taste)

- Sawayaka (flinty aftertaste)

- Kire (no aftertaste)

Next, sake tasting was carried out by 101 evaluators who were randomly selected from among employees of Ozeki Corporation (hereafter referred to as panelists).

Sake tasting of four sets of sake samples, each consisting of four (including replicates) of 10 sake samples, was carried out for four days. Samples E and F, whose data are plotted at the center of the scatter diagram, were commonly selected. Two other samples (e.g., samples A and

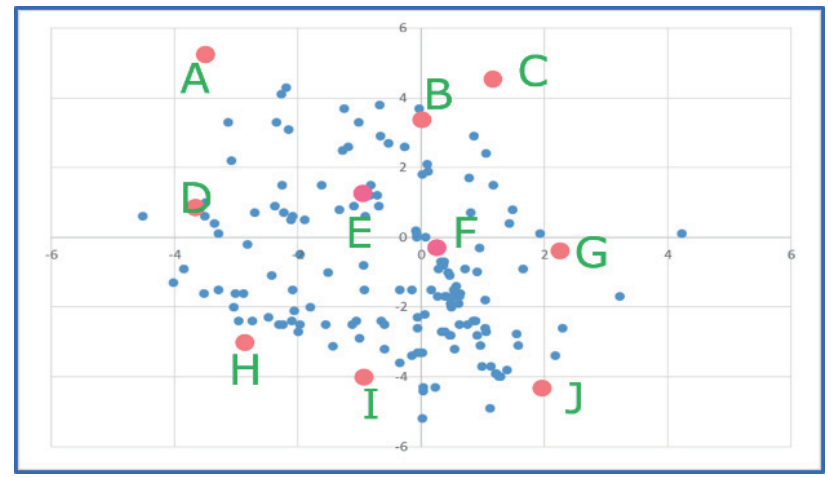

Fig. 2. (Color online) Position of sake sample extracted from two-dimensional scatter plot. 
J, samples B and I, samples C and H, and samples D and G) whose data are largely scattered on the scatter diagram were added to samples $\mathrm{E}$ and $\mathrm{F}$. This is because precise evaluation becomes difficult for panelists who are required to taste many samples at once owing to the effects of alcohol. In addition, to avoid the effects of order and labels in the sensory evaluation, each sample in each set was randomly coded so that the panelists could start tasting different samples. ${ }^{(14)}$ The panelists were asked to select multiple words from among 13 evaluation words that they think appropriately express the taste of each sample. During sensory evaluation, $100 \mathrm{ml}$ of sample is poured into amber glasses at room temperature $\left(20^{\circ} \mathrm{C}\right)$, each panelist holds a sample of about $5 \mathrm{ml}$ in the mouth to evaluate taste and aroma.

\subsection{Statistical analysis}

The relationship between values obtained using the taste sensing system and evaluation words and that amongst evaluation words were statistically analyzed on the basis of the results of the sensory evaluation.

The frequency of selection of each evaluation word by panelists are summarized in Table 5 . The results indicate that the selection frequencies of evaluation words differ among the samples and that frequently selected evaluation words indicate the characteristics of the flavor of each sample.

Next, the tendency of the evaluation words selected by panelists was analyzed by cluster analysis (Fig. 3). It was found that the 13 evaluation words are classified into four clusters that represent four different flavors. For the cluster analysis, a hierarchical method such as the nearest-neighbor method, without the use of the statistical analysis software, statistical package for social science (SPSS), was adopted. ${ }^{(15)}$

Table 5

Results of sensory evaluation.

\begin{tabular}{|c|c|c|c|c|c|c|c|c|c|c|}
\hline & $\mathrm{A}$ & $\mathrm{B}$ & $\mathrm{C}$ & $\mathrm{D}$ & E & $\mathrm{F}$ & $\mathrm{G}$ & $\mathrm{H}$ & I & $\mathrm{J}$ \\
\hline Marumi & 0.00 & 0.13 & 0.04 & 0.16 & 0.10 & 0.35 & 0.12 & 0.15 & 0.25 & 0.13 \\
\hline Fukurami & 0.00 & 0.13 & 0.04 & 0.04 & 0.07 & 0.23 & 0.36 & 0.15 & 0.21 & 0.25 \\
\hline Koku & 0.13 & 0.21 & 0.26 & 0.00 & 0.20 & 0.16 & 0.40 & 0.15 & 0.33 & 0.38 \\
\hline Atsumi & 0.00 & 0.17 & 0.15 & 0.00 & 0.08 & 0.17 & 0.20 & 0.15 & 0.46 & 0.25 \\
\hline Sakiaji & 0.00 & 0.25 & 0.30 & 0.00 & 0.08 & 0.07 & 0.24 & 0.15 & 0.13 & 0.17 \\
\hline Odayaka & 0.00 & 0.08 & 0.04 & 0.32 & 0.18 & 0.28 & 0.08 & 0.07 & 0.17 & 0.08 \\
\hline Atoaji & 0.13 & 0.17 & 0.26 & 0.00 & 0.20 & 0.21 & 0.28 & 0.11 & 0.33 & 0.29 \\
\hline Soft & 0.13 & 0.17 & 0.15 & 0.20 & 0.07 & 0.21 & 0.00 & 0.07 & 0.04 & 0.08 \\
\hline Sarari & 0.46 & 0.13 & 0.07 & 0.40 & 0.16 & 0.07 & 0.00 & 0.37 & 0.04 & 0.00 \\
\hline Sukkiri & 0.50 & 0.17 & 0.19 & 0.40 & 0.30 & 0.15 & 0.08 & 0.15 & 0.13 & 0.08 \\
\hline Sawayaka & 0.04 & 0.17 & 0.19 & 0.12 & 0.10 & 0.04 & 0.16 & 0.15 & 0.04 & 0.13 \\
\hline Keikai & 0.38 & 0.13 & 0.22 & 0.44 & 0.19 & 0.05 & 0.04 & 0.11 & 0.08 & 0.00 \\
\hline Kire & 0.21 & 0.21 & 0.30 & 0.08 & 0.21 & 0.09 & 0.04 & 0.19 & 0.08 & 0.08 \\
\hline
\end{tabular}

Ordinate: Sensory evaluation word

Abscissa: Samples A-H

For each sample, selection frequencies of sensory evaluation words by panelists are calculated. 


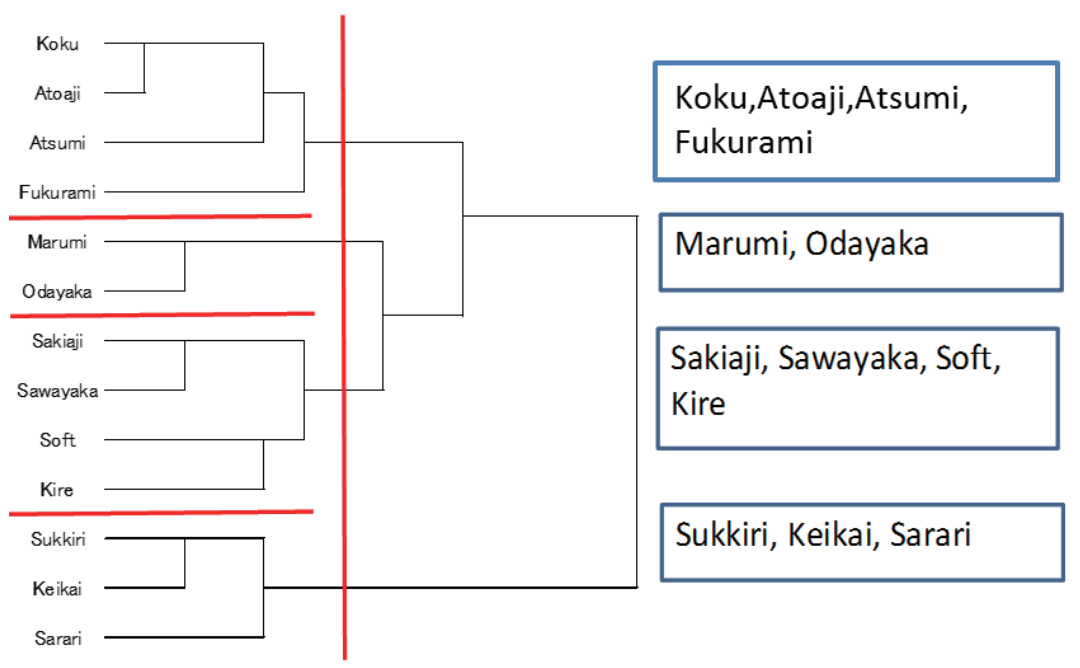

Fig. 3. (Color online) Results of cluster analysis.

On the two-dimensional scatter diagram, the evaluation words that were frequently selected by the panelists from among the 10 candidates were superimposed, as shown in Fig. 4, and then classified (by color) into four clusters on the basis of the results of the cluster analysis. As a result, the "Koku", "Atoaji", "Atsumi", and "Fukuami" in the cluster in Fig. 3 were found to fall on the right side of the scatter diagram. Similarly, "Marumi" and "Odayaka" fell on the lower side, "Sakiaji", "Sawayaka", "Soft", and "Kire" on the upper side, and "Sukkiri", "Keikai", and "Sarari" on the left side.

From the above results, the samples that were judged to have similar tastes by the sensory evaluation were considered to be closely plotted on the scatter diagram. Thus, the scatter diagram was divided into four areas corresponding to the cluster analysis results in Fig. 3 on the basis of the results of the sensory evaluation of taste.

\subsection{Development of sake flavor chart}

Evaluation words that correspond to each cluster were allocated into the four areas on the scatter diagram. However, the purpose of this study, i.e., the visualization of sake flavor, was not achieved because there were too many words to express the taste represented in each area.

To represent the 13 evaluation words more simply, sensory evaluation by six trained panelists was carried out again to determine the words that can best express the taste of the samples in the four areas on the scatter diagram in Figs. 3 and 4. The four areas were described as "rich", "refreshing", "crisp", and "deep", and the axes dividing the areas were described as "light/rich" and "initial taste/aftertaste". In addition, on the basis of discussion among the trained panelists, the sake samples plotted at the center of the scatter diagram were found to have a good balance of the four tastes. Therefore, a fifth area described as "mild" was added at the center of the scatter diagram. Figure 5 shows the thus-obtained scatter diagram, which we called the sake flavor chart. 


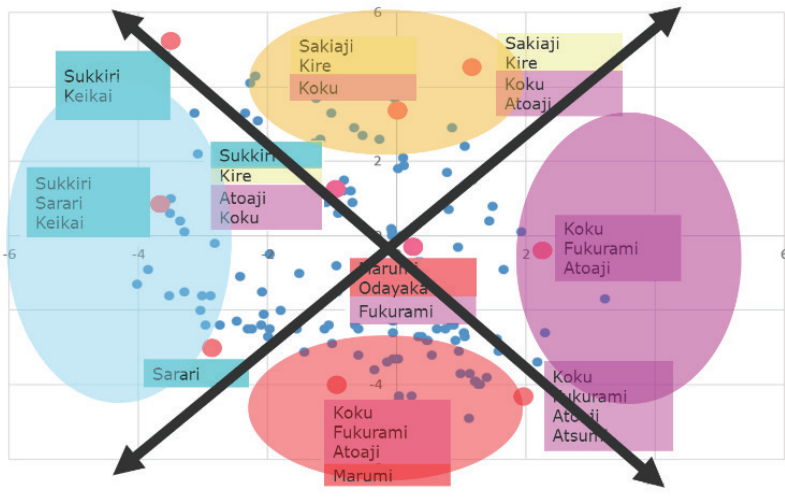

Fig. 4. (Color online) Classification of sake tastes in two-dimensional scatter diagram.

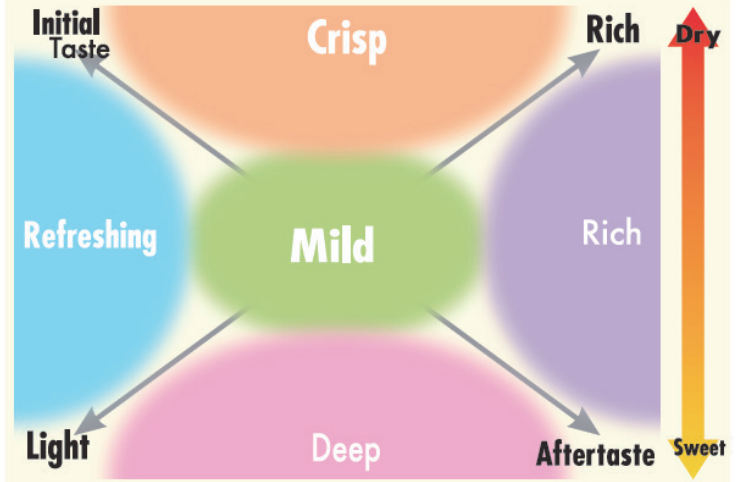

Fig. 5. (Color online) Sake flavor chart.

\section{Conclusions}

By analyzing sake by the taste sensor and by combining its value with the general analysis value and the sensory evaluation result using a statistical method, it was possible to visualize the taste of sake objectively with little divergence from the sensory evaluation.

The sake flavor chart developed in this study is a two-dimensional chart based on the measurement results obtained using only analytical devices. The meaning of the representation based on the sensory evaluation became apparent after the data of sake samples were mapped on the chart. Therefore, the lack of objectivity in the conventional method, which has presented a challenge in the use of conventional two-dimensional scatter diagrams, was solved because the chart can be developed using only the results obtained with analytical devices. In addition, by defining the areas and axes that represent the taste of sake using a common standard based on the detailed results of sensory evaluation, a shared perception of taste among panelists became possible, eliminating the differences between the evaluations made by panelists in actual sake tasting and those derived from charts.

From the above, providing a common objective standard of sake taste to general consumers will become possible using the sake flavor chart. We hope that sake taste will become more understandable and friendly to consumers by using the sake flavor chart as the new standard.

The current sake flavor chart, however, does not consider the aromatic components of sake, which are also important in addition to taste. This will be left for future studies. In addition, the compatibility between sake and food will be examined in the future, because sake is frequently enjoyed with meals.

\section{References}

1 K. Iwano: Brewing Technology of Sake, Revised Edition (Brewing Society of Japan, Tokyo, 1998) (in Japanese).

2 N. Nishitani, Ed.: Prescription Method of National Tax Agency (Brewing Society of Japan, Tokyo, 1974) (in Japanese). 
3 S. Sato, H. Kawashima, and Y. Maruyama: J. Brew. Soc. Jpn. 69 (1974) 774 (in Japanese).

4 Sake Service Institute: Basics of Sake - for Professionals of Sake-, Textbook (Sake Service Institute, Tokyo, 2009) p. 110 (in Japanese).

5 National Tax Agency: Study on the Sound Development of Sake-Brewing Industry, http://www.nta.go.jp/ shiraberu/senmonjoho/sake/kasseika/hokoku/01.htm (accessed November 2005) (in Japanese).

6 S. Nakayama H. Saito, H. Sakurai, H. Nakai, and H. Ikezaki: Iwate Industrial Research Institute Res. Rep. 2 (1995) 97 (in Japanese).

7 S. Iiyama, T. Ikeda, K. Toko, and M. Yahiro: J. Jpn. Soc. Food Sci. Tech. 44 (1997) 615 (in Japanese).

8 N. Nakahara, H. Sakaida, T. Kai, Y. Sakakibara, K. Nishiyama, N. Fukuda, and M. Suiko: J. Jpn. Soc. Food Sci. Tech. 52 (2005) 145 (in Japanese).

9 K. Otubo, H. Ikezaki, A. Taniguchi, H. Okadome, H. Toyoshima, and T. Inbe: IEEE Tech. Rep., OME 2000-62 (2000).

10 K. Toko: Molecular Recognition of Taste and Smell (The Chemical Society of Japan, Tokyo, 2002) pp. 113-121 (in Japanese).

11 K. Toko: Sensory Biosensor (Asakura Publishing Co., Ltd., Tokyo, 2002) pp. 130-185 (in Japanese).

12 K. Toko: Science of Taste (Kadokawa Corporation, Tokyo, 2002) pp. 145-197 (in Japanese).

13 H. Stone, J. Sidle, S. Oliver, A. Woolsey, and R. C. Singleton: Food Technol. 28 (1974) 11.

14 Japanese Society for Sensory Evaluation: Textbook for Professional Sensory Panelists (Kenpakusha, Tokyo, 2009) (in Japanese).

15 Y. Tanaka and K. Wakimoto: Methods of Multivariate Statistical Analysis (Gendai-Sugakusha, Kyoto, 1983) (in Japanese).

\section{About the Authors}

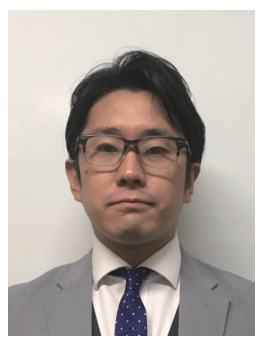

Yoichiro Kanno received his B.S. degree from Kyoto University, Japan, in 2009 and his M.S. degree from Hiroshima University, Japan, in 20011. From 2011 to 2016, he was a researcher and since 2016, he has been a product developer at Ozeki Corporation, Japan. His research interests are in fermentation, statistics, and biotechnology.

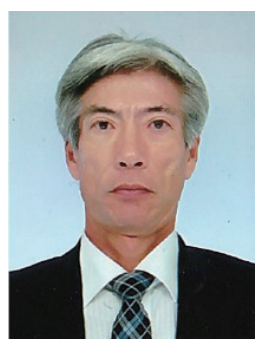

Toshitaka Minetoki received his B.S. and M.S. degrees from Ehime University, Japan, in 1984 and 1986, respectively, and his Ph.D. degree from the University of Tokyo, Japan, in 1999. Since 1986, he has been at Ozeki Corporation. His research interests are in bioscience and biotechnology.

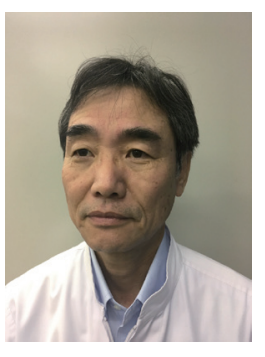

Takayuki Bogaki received his B.S. and M.S. degrees from Tottori University, Japan, in 1987 and 1989, respectively, his M.B.A. degree from Kwansei Gakuin University, Japan, in 2005, and his Ph.D. degree from Kanazawa Institute of Technology, Japan, in 2017. From 1989 to 2011, he was a researcher, and since 2011, he has been a general manager at the General Research Institute of Ozeki Corporation, Japan. His research interests are in fermentation, food engineering, and biotechnology. 


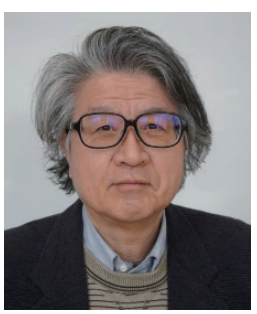

Kiyoshi Toko received his B.E., M.E., and Ph.D. degrees from Kyushu University, Japan, in 1975, 1977, and 1982, respectively. He was the Dean of the Graduate School of Information Science and Electrical Engineering, Kyushu University, from 2008 to 2011, and he is currently a Distinguished Professor. His research interests are in Kansei biosensors, especially taste sensors and odor sensors. 